EPJ Web of Conferences 116, 02001 (2016)

DOI: $10.1051 /$ epjconf/201611602001

(C) Owned by the authors, published by EDP Sciences, 2016

\title{
Energy reconstruction of high energy muon and neutrino events in $\mathrm{KM} 3 \mathrm{NeT}$
}

\author{
Evangelia Drakopoulou $^{1,2, a}$, Christos Markou ${ }^{1, \mathrm{~b}}$, Ekaterini Tzamariudaki ${ }^{1, \mathrm{c}}$, Konstantinos Pikounis $^{1,2, \mathrm{~d}}$ \\ for the KM3NeT Collaboration \\ ${ }^{1}$ N.C.S.R. Demokritos, Patriarchou Grigoriou and Neapoleos 27, Agia Paraskevi, Greece \\ ${ }^{2}$ National Technical University of Athens, Heroon Polytechniou 9, Zografou Campus, Greece
}

\begin{abstract}
KM3NeT will be a European deep-sea infrastructure of neutrino telescopes covering a volume of several cubic kilometers in the Mediterranean Sea aiming to search for high energy neutrinos from galactic and extragalactic sources. This analysis focuses on muons coming from neutrino charged-current interactions. In large water Cherenkov detectors the reconstructed muon is used to approximate the neutrino direction and energy, thus providing information on the astrophysical neutrino source. Muon energy estimation is also critical for the differentiation of neutrinos originating from astrophysical sources from neutrinos generated in the atmosphere which constitute the detector background. We describe a method to determine the muon and neutrino energy employing a Neural Network. An energy resolution of approximately 0.27 has been achieved for muons at the $\mathrm{TeV}$ range.
\end{abstract}

\section{Introduction}

The KM3NeT Collaboration aims to deploy a neutrino telescope with volume of several cubic kilometers at the Mediterranean Sea that will search for neutrinos from galactic and extragalactic sources. The first phase of the neutrino telescope is called the KM3NeT-ARCA detector and consists of two building blocks covering a total volume of approximately $1 \mathrm{~km}^{3}$. The basic detection units are the optical modules (OMs) consisting of 17 inch diameter pressure-resistant glass spheres, each equipped with 31 photomultipliers (PMTs) of 3-inch photocathode diameter and their readout electronics. The PMTs are arranged in vertical strings covering a roughly cylindrical instrumented volume.

The reconstruction of the muon and consequently the neutrino energy is critical for neutrino telescopes. The signal corresponds to astrophysical $v_{\mu}$ events with an energy spectrum extending to higher energies compared to the energy spectrum of the atmospheric $v_{\mu}$ events which are the background. The energy estimation thus allows the discrimination of astrophysical $v_{\mu}$ from atmospheric $v_{\mu}$ events that refer to neutrinos produced by charged Kaons or pions during the interaction of

\footnotetext{
a e-mail: liliadrak@inp.demokritos.gr

b e-mail: cmarkou@inp.demokritos.gr

c e-mail: katerina@inp.demokritos.gr

d e-mail: pikounis@inp.demokritos.gr
}

This is an Open Access article distributed under the terms of the Creative Commons Attribution License 4.0, which permits unrestricted use, distribution, and reproduction in any medium, provided the original work is properly cited. 
cosmic rays in the atmosphere. Moreover, the determination of the energy is a major parameter in the identification of the neutrino sources as it enhances many analyses performed in Cherenkov detectors, such as searches for point sources of neutrinos or diffuse extraterrestrial neutrinos.

Monte-Carlo simulations have been performed for one block (with a volume of $\simeq 0.5 \mathrm{~km}^{3}$ ) of the KM3NeT-ARCA detector including the neutrino interactions in the medium, generation and propagation of secondary particles, the light generation and propagation in water and finally the PMT response to the incident photons. The light from the background of ${ }^{40} \mathrm{~K}$ decays is also considered. $10^{10}$ neutrinos have been generated according to a spectrum $E^{-1.4}$, instead of $E^{-2}$ that is theoretically expected, in order to get reasonable statistics in the whole energy range from $10^{2} \mathrm{GeV}$ to $10^{8} \mathrm{GeV}$. A cylindrical volume, the can, encloses the detector volume and is used for the simulation of the Cherenkov photons. The radius and the height of the can is approximately three times the absorption length of light in sea water larger than the detector volume. A larger volume, the generation volume, is used for neutrino generation and corresponds to the can volume expanded at the maximum lepton range in the medium (rock or sea water) for the maximum value of the energy range that is generated. Prior to the estimation of energy, the direction of the particle is reconstructed using the standard algorithm for track reconstruction in KM3NeT. In the final event sample, events are selected (well reconstructed tracks) if the maximum $\log$-likelihood value per degree of freedom found by the fit $(\Lambda)$ satisfies the relation $\Lambda>-5.8$ [1].

\section{Energy reconstruction using a neural network}

Muons lose energy via ionization and by stochastic processes, such as bremsstrahlung, pair production, and photonuclear interactions. The total average energy loss of the muon is:

$$
\frac{-d E}{d x}=a(E)+b(E) \cdot E_{\mu}
$$

where $a \simeq 0.274 \mathrm{GeV} \mathrm{m}^{-1}$ accounts for the energy loss due to ionization and $b \simeq 0.000349 \mathrm{~m}^{-1}$ is due to the stochastic energy loss. Muons with $E_{\mu}>1 \mathrm{TeV}$ lose energy dominantly stochastically, while for lower energies ionization dominates. In this study a method to derive muon (and neutrino energy) from the light collected as the muon passes through the instrumented volume has been developed.

Since the reconstruction of the muon energy is based on the collection of light in PMTs, muons should travel an adequate distance inside the instrumented volume before an attempt to estimate the energy is made. Therefore, a containment selection has been established aiming to distinguish muon tracks which are well contained in the detector, thus depositing the light in the instrumented volume, from muons that pass outside or cross the borders of the detector. Muons which do not travel an adequate distance inside the detector deposit only a fraction of light in the instrumented volume thus not allowing a reliable estimation of the muon energy. In this case a lower limit of the muon energy is calculated.

This method employs a Multi-Layer Percepton Neural Network with appropriate input variables that show a strong dependence on the muon energy and can thus be used for the energy reconstruction. These variables are:

i. The number of optical modules with hits used for the track direction reconstruction per track length.

ii. The number of photomultipliers with recorded hits $\left(N_{P M T S}\right)$ that are used for the track reconstruction, weighted according to the vertical distance from the reconstructed track $\left(D_{i}\right)$. This weight refers to the ratio $D_{i} / D_{\max }$ where $D_{\max }$ is a maximum distance of $500 \mathrm{~m}$.

iii. The ratio of $N_{P M T S}$ to the number of PMTs that could have been hit by Cherenkov photons according to the direction of the reconstructed track but have not recorded pulses.

iv. The total Time over Threshold (ToT) (as a measure of charge) of all PMTs that are used for the track reconstruction. 

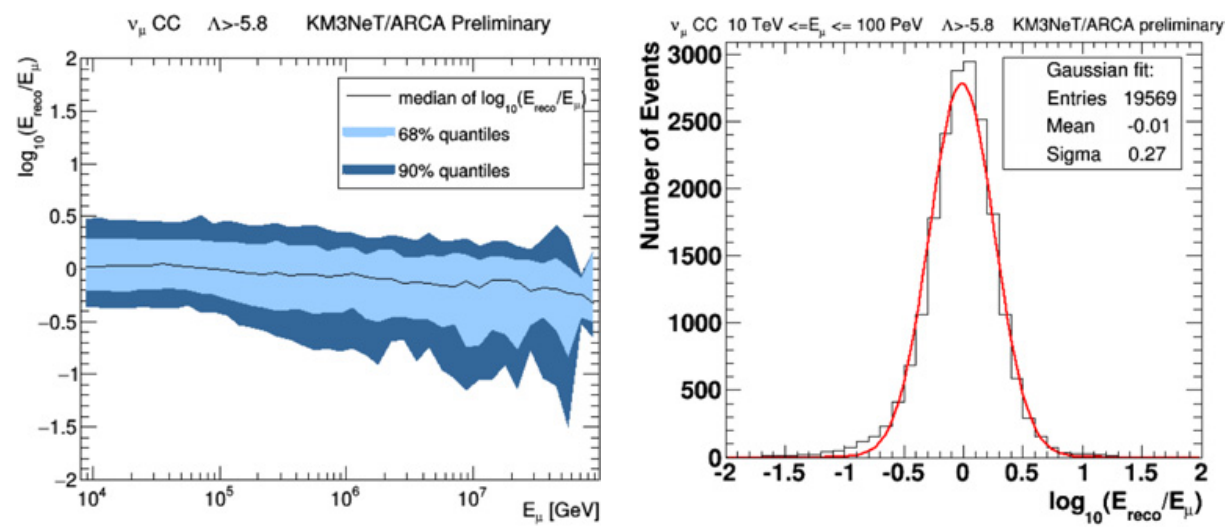

Figure 1. Left plot: The median of $\log _{10}\left(E_{\text {reco }} / E_{\mu}\right.$ ) (with $68 \%$ and $90 \%$ quantiles) as a function of $E_{\mu}$ for events that satisfy the containment selection. Right plot: The distribution of $\log _{10}\left(E_{\text {reco }} / E_{\mu}\right)$ for events with $E_{\mu} \geq 10 \mathrm{TeV}$ that satisfy the containment selection. A Gaussian fit is applied.

The input variables are inserted in the Neural Network which is trained and tested using half the sample of $v_{\mu}$ events while the energy is determined for the other half of events to avoid bias.

\subsection{Results on muon and neutrino energy reconstruction}

The results on the muon energy reconstruction for reconstructed events with $\Lambda>-5.8$ that satisfy the containment selection are shown in Fig. 1. Figure 1 (left plot) depicts the median of $\log _{10}\left(E_{\text {reco }} / E_{\mu}\right)$ with $68 \%$ and $90 \%$ quantiles as a function of $E_{\mu}$ where $E_{\mu}$ is the MC muon energy at the can level and $E_{\text {reco }}$ is the reconstructed muon energy. A very good energy reconstruction has been achieved for high energy muons with $10 \mathrm{TeV} \leq E_{\mu} \leq 100 \mathrm{PeV}$ resulting in a median of 0.02 for $E_{\mu} \geq 10 \mathrm{TeV}$ while for muons with energies $E_{\mu}>10 \mathrm{PeV}$ the calculation suffers from limited statistics. The energy resolution that has been attained for these events, that correspond to approximately $90 \%$ of all the reconstructed events with $\Lambda>-5.8$, is $\simeq 0.27$ in $\log _{10} E_{\mu}$ as it is shown in Fig. 1 (right plot). For the rest $10 \%$ of events that do not satisfy the containment selection a lower limit of the energy is calculated. In this case the energy is underestimated leading to larger tails in the left side of the distribution of $\log _{10}\left(E_{\text {reco }} / E_{\mu}\right)$ for all reconstructed events. However, due to the low percentage of these events $(10 \%)$ in the whole sample, the energy resolution is $\simeq 0.28$.

The neutrino energy cannot be reliably reconstructed for events that cross the detector and the neutrino interaction vertex lies outside the instrumented volume. In such cases most photons from the hadronic activity are not detected, thus resulting in an underestimation of the neutrino energy. Only a lower limit of the neutrino energy can then be reported that corresponds to the reconstructed muon energy. In order to make sure that the muon and neutrino energy is reliably reconstructed for events with the neutrino vertex close or inside the instrumented volume we consider events with the reconstructed vertex inside a fiducial volume which is slightly smaller than the volume of the detector. This criterion alone is not sufficient to reject events that have interacted outside the detector. Therefore we apply selection criteria originally developed for atmospheric muon rejection. This selection keeps most events that have interacted inside this fiducial volume and rejects events with high activity on border strings. Moreover, events with activity upstream of the reconstructed vertex that is compatible with the track hypothesis are examined and rejected if they have given pulses in PMTs at the edge of the detector. The results on muon and neutrino energy reconstruction (obtained using the aforementioned Neural Network) for this subsample of events, are shown in Fig. 2 (left and right plot respectively). 

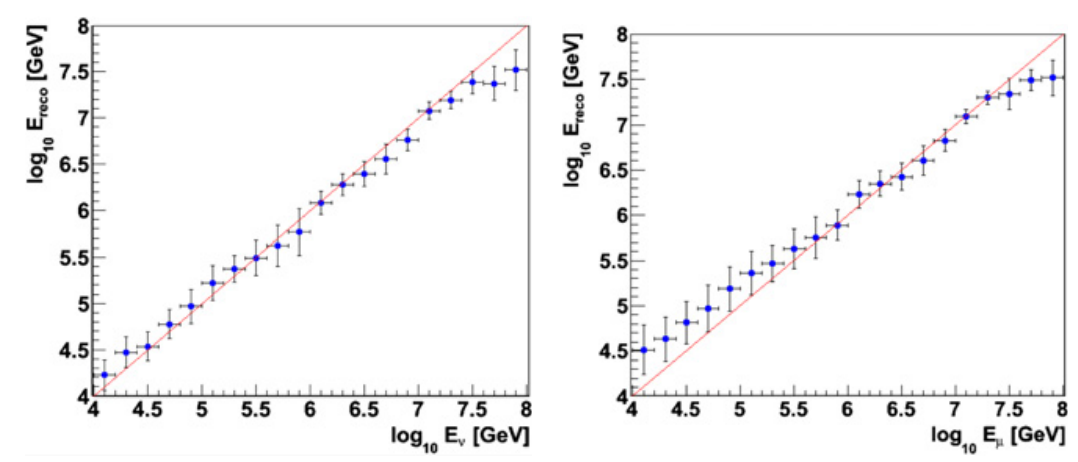

Figure 2. Left (right) plot: The reconstructed energy $\left(\log _{10} E_{\text {reco }}\right)$ with respect to the muon (neutrino) energy for events (with $E_{\mu} \geq 10 \mathrm{TeV}$ ) that satisfy the containment selection and the interaction vertex is estimated to be inside the fiducial volume. The mean with RMS error is depicted.

The overestimation of the muon energy (left plot) is due to the fact that most photons from the hadronic shower are detected. A very good linear relation between the reconstructed energy with the neutrino energy can be observed (right plot) leading to a reliable reconstruction of the neutrino energy for tracks that interact well inside the instrumented volume.

The systematics uncertainties for this energy reconstruction have been studied assuming a variation of $\pm 10 \%$ on the estimation of the absorption and scattering length of light in sea water and on the effective area of the PMTs. The relative deviation in the mean reconstructed energy $\Delta E / E$ corresponds to $\pm 3 \%, \pm 0.1 \%$ and $\pm 1 \%$ respectively.

This method for the muon energy reconstruction has been applied to an alternative detector configuration with a larger average horizontal distance between the detection units (strings) leading to an instrumented volume of $\simeq 0.8 \mathrm{~km}^{3}$ (compared to $\simeq 0.5 \mathrm{~km}^{3}$ for the previous detector configuration). The containment selection and the input variables have been appropriately adjusted in this case and the Neural Network has been trained again. For high energy events with $10 \mathrm{TeV} \leq E_{\mu} \leq 100 \mathrm{PeV}$ satisfying the containment selection an energy resolution of $\simeq 0.25$ in $\log _{10} E_{\mu}$ has been achieved increasing to $\simeq 0.26$ when all reconstructed events with $\Lambda>-5.8$ are taken into account.

\section{Conclusions}

The presented work has been partially financed by the European Social Fund (NSRF 2007-2013) under the Thalis initiative GRBNeT project-MIS 360381. A method to estimate the muon and neutrino energy in the KM3NeT-ARCA detector, has been presented. A very good energy resolution of $\simeq 0.27$ in $\log _{10} E_{\mu}$ for high energy muons with $10 \mathrm{TeV} \leq E_{\mu} \leq 100 \mathrm{PeV}$ has been attained which improves to $\simeq 0.25$ in $\log _{10} E_{\mu}$ when a larger instrumented volume is considered.

\section{Reference}

[1] A. Trovato, E. Drakopoulou, P. Sapienza, proceedings of ICRC2015 (2015), 8, to be published 